\title{
Scheduling of Twin Telescopes and the Impact on Troposphere and UT1 Estimation
}

\author{
A. Corbin* \\ Institute for Geodesy and Geoinformation, University of \\ Bonn \\ R. Haas $^{\dagger}$ \\ Chalmers University of Technology, Department of Space, \\ Earth and Environment, Onsala Space Observatory
}

December 13, 2019

This paper is a non-peer reviewed preprint submitted to EarthArXiv. It will pe published in the proceedings of the $24^{\text {th }}$ Meeting of the European VLBI Group for Geodesy and Astrometry with the citation key:

R. Haas, S. Garcia-Espada, and J. A. Lopez Fernandez (eds.) 'Proceedings of the 24th European VLBI Group for Geodesy and Astrometry Working Meeting', DOI: 10.7419/162.08.2019, ISBN: 978-84-416-5634-5

*mail: corbin@geod.uni-bonn.de; Corresponding author

†mail: rudiger.haas@chalmers.se 


\section{Scheduling of twin telescopes and the impact on troposphere and UT1 estimation}

Armin Corbin and Rüdiger Haas

\begin{abstract}
Recently, several VGOS twin telescopes in Europe were completed. We examine the use of VGOS twin telescopes by a new scheduling approach. This approach is based on integer linear programming and creates uniform distributed observations over time. Several VLBI intensive sessions are rescheduled involving the VGOS twin telescopes and the impact on the troposphere and UT1 estimation is investigated.
\end{abstract}

Keywords VLBI Intensive Sessions; VGOS Twin Telescopes; Scheduling; Simulation; Atmospheric Turbulence; Global Optimization

\section{Introduction}

VLBI intensive (INT) sessions (Schnell, 2006) are one hourly sessions that are used to provide information about the solar time UT1 on a regular basis and with low latency. From Monday to Friday the INT1 baseline from Wettzell to Kokee is observed and on the weekend the INT2 baseline from Wettzell to Ishioka. These baselines have large east-west components ensuring a high sensitivity to the solar time UT1. See Fig. 1 for the location of the telescopes. Currently, the legacy S/X setup is used for all INT sessions, however, at each of the stations involved in the INT1 and INT2 sessions a modern VGOS-compatible telescope is available. Thus, it is possible to observe broadband INT sessions. Moreover, there are VGOS twin tele-

Armin Corbin, Rüdiger Haas

Department of Space, Earth and Environment, Chalmers University of Technology, Observatorievägen 90, SE-439 92 Onsala, Sweden

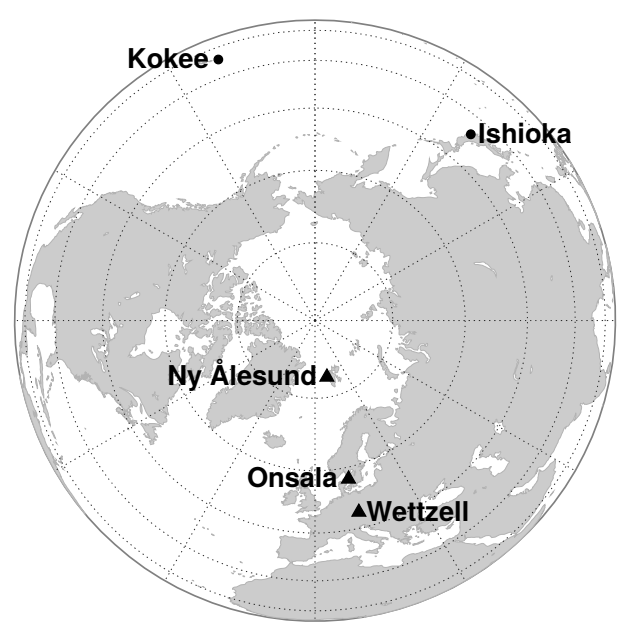

Fig. 1: Map of the northern hemisphere showing the position of all observatories with VGOS twin telescopes (triangles) and observatories with one only VGOS telescope used for the INT1 and INT2 sessions (circles).

scopes at Wettzell enabling simultaneous observation of the INT1 and INT2 baselines. In total there are three twin telescopes on the globe, which are all located in Europe (Fig 1). Involving both the VGOS twin telescopes at Wettzell and Onsala, as well as the VGOS telescopes at Kokee and Ishioka, four baselines with large east-west component can be observed simultaneously.

We created new schedules for 187 INT sessions of the year 2018. For each of these sessions three different schedules were created. One without twin telescopes, one with the twin telescopes in Wettzell and another one with the twin telescopes in Wettzell and Onsala. We used a new scheduling approach capable of finding 
the schedule with the best sky coverage score (Sec. 2). In the next step, the observations of each schedule were simulated (Sec. 3). The simulator ensures that the atmosphere and the clocks for each session are independent of the schedule. Finally, the simulated observations were adjusted and the estimated parameters were compared (Sec. 4). All computations were done with the VLBI analysis toolbox ivg::Ascot (Halsig et al., 2017).

\section{Scheduling}

For each investigated INT session three different schedules were created. The main difference between them is the number of involved twin telescope stations. Thus, we call the scenarios ZT (zero twin), OT (one twin), and TT (two twins).

In the $Z T$ scenario the same observatories as in the original INT schedules are used, but with the VGOS instead of the S/X legacy telescopes.

In the $O T$ scenario the INT1 and INT2 baseline are observed simultaneously with the VGOS telescopes in Kokee and Ishoika and the VGOS twin telescopes in Wettzell.

Finally, in the $T T$ scenario the VGOS twin telescopes in Wettzell and Onsala as well as the VGOS telescopes in Ishioka and Kokee are used. In this scenario four baselines with large east-west component are observed simultaneously. To improve the sky coverage we also observe the short baselines between the twin telescopes in Onsala and Wettzell and the baseline between Ishioka and Kokee every fourth minute. The detailed scan sequence is presented in Tab. 1.

We created schedules with regular observations: In each minute each station is participating in one quasar observation. The first $30 \mathrm{~s}$ are reserved for the slewing of the telescope and the remaining $30 \mathrm{~s}$ are used for the observation. This is possible because VGOS telescope are very fast, i.e., they can slew to any source on the sky within $30 \mathrm{~s}$. Only observations exceeding a signal to noise ratio (SNR) larger than 20 within the $30 \mathrm{~s}$ were scheduled. For the SNR computation (e.g. Gipson 2018) we assumed a VGOS broadband setup with 32 channels of $32 \mathrm{MHz}$ bandwidth each and 2bit sampling. However, we only used one polarization, leading to a pessimistic SNR compared with the VGOS setup. Moreover, the antennas of a twin tele-
Table 1: Overview of the schedule setups. We use the IVS 2-letter code to identify the stations. A baseline or subnet is a group of 2-letter codes and different subnets are divided by white spaces.

\begin{tabular}{|c|c|c|c|c|c|}
\hline \multicolumn{2}{|c|}{ scenario|scan sequence } & \multicolumn{4}{|c|}{ |\#obs. \#scans \#stas. \#long bl. } \\
\hline$Z T$ & $\begin{array}{l}\text { WnK2 (INT1) } \\
\text { WnIs (INT2) }\end{array}$ & 60 & 60 & 2 & 1 \\
\hline$O T$ & $\mid \begin{array}{ll}3 \mathrm{x} \text { WnK2 } & \text { WsIs } \\
3 \mathrm{x} \text { WnIs } & \text { WsK2 }\end{array}$ & 120 & 120 & 4 & 2 \\
\hline$T T$ & $\left|\begin{array}{l}3 x \text { WnOeK2 WsOwIs } \\
1 x \text { K2Is WsOw WnOe } \\
3 x \text { WnOeIs WsOwK2 } \\
1 x \text { K2Is WsOw WnOe }\end{array}\right|$ & 315 & 135 & 6 & 4 \\
\hline
\end{tabular}

scope were never observing the same source together. In fact, the spherical distance between the simultaneously observed sources of a twin station had to be larger than $60^{\circ}$. At least 10 minutes had to past until the same source was observed again by the same station. Moreover, a source could not be observed more than 6 times by the same observatory (observations of a twin telescopes are not counted separately).

The optimization criterion for the scheduling is the local sky coverage. We used the sky coverage score described in Corbin et al. (2019): The hemisphere above each station is partitioned into cells of equal size and similar shape multiple times. If a cell includes an observation its surface area is added to the sky coverage score. The score is computed for a time period of 10 minutes which is shifted in 5 minutes steps. The partitions are computed following Beckers \& Beckers (2012) and have 10, 18, 29 and 107 cells. Twin telescopes were treated as a single telescope for the computation of the sky coverage.

We used an integer linear program (ILP, Williams 2013) that incorporates all the constraints explained above to create the schedules. The ILP is descried in detail in Corbin et al. (2019). However, the ILP we are using in this paper is extended with additional constraints to model the twin stations. Since the observation times and the subnets were predefined (see Tab. 1) the ILP's only task was to determine the source that was observed by each subnet. With these simplifications it is possible to find the schedule with the optimal sky coverage in a few minutes using a CPU with 2 cores at 2,7 GHz. 


\section{Simulations}

To simulate an observation $\tau_{\text {sim }}$ we computed the geometric delay $\tau_{g}$ and distorted it with systematic and random errors:

$$
\begin{aligned}
\tau_{\text {sim }}=\tau_{g} & + \text { clock }_{2}-\text { clock }_{1} \\
& +M f\left(\varepsilon_{2}\right) \cdot \mathrm{ZWD}_{2}-M f\left(\varepsilon_{1}\right) \cdot \mathrm{ZWD}_{1}+\mathrm{wn}
\end{aligned}
$$

For each station $i$ we modeled the clock clock $_{i}$ and the delay caused by the wet part of the troposphere in zenith direction $\mathrm{ZWD}_{i}$, which was mapped to the elevation $\varepsilon_{i}$ of the observation with a mapping function $M f$. Here, we used the VMF1 mapping function (Böhm et al., 2006). To each observation, white noise with a standard deviation of 20 ps was added to simulate random errors.

The geometric delay $\tau_{g}$ was calculated according to the IERS conventions (Petit \& Luzum, 2010). We used the ITRF2014 (Altamimi et al., 2016) for station coordinates and the ICRF2 (Fey et al., 2015) for source positions. The IERS C04 time series was used for nutation and polar motion. However, we used the BKG eopi time series for UT1, to reduce the interpolation error.

The geometric delay is the only deterministic term in Eq. 1. The other terms are stochastic and require a random generator for the computation. Each session was simulated three times, each time with a different schedule. To avoid different clocks for each simulation of the same session, the station clocks were precomputed and saved. We assumed an Allan standard deviation of 10 fs at 50 minutes. During the simulation the corresponding clock value was looked up and added to the delay. With this method, the station clocks are consistent for each run of the simulation and independent of the schedule.

To make sure that in each simulation of the same session and station the same troposphere was used, a reference troposphere was computed. To do so, the sky above each station was partitioned into 209 cells of equal size and similar shape using the method from Beckers \& Beckers (2012). For each cell the equivalent zenith wet delay (EZWD) was computed with a temporal resolution of one minute. The EZWDs are calculated with the turbulence model from Nilsson \& Haas (2010) using an initial delay of 450 ps. We used the turbulence parameters provided in Petrachenko et al. (2009), except for the station Onsala that is missing in the list.
During the simulation the EZWD from the cell containing the observation was mapped to the correct elevation and added to the simulated delay. For twin stations the same reference troposphere was used. See Fig. 2 for an illustration of the reference troposphere.

To verify the simulator we simulated the observations of 114 INT sessions in the year 2018. Only sessions with more than 15 good $^{1}$ observations were used. Afterwards, the simulated as well as the observed sessions were analyzed using a least-squares adjustment with standard intensive parametrization ${ }^{2}$. For each session we computed the difference in UT1 between the solution using the simulated observations and the solution using the real observations. The WRMS of these differences is $14 \mu$ s which indicates that the simulated observations are reasonable.

\section{Results}

The simulated sessions were evaluated with a leastsquares adjustment. Due to the large number of observations (Tab. 1), more parameters can be estimated than in regular INT sessions. Instead of one ZWD offset we approximated constant piece wise linear functions (CPWLF) with an interval length of 10 minutes (7 parameters per station). The station clocks were modeled with a CPWLF with 20 minutes interval length (4 parameters per station). The clock at WETTZ13N was fixed. The target parameter was UT1. We used two different parametrizations for the ZWD for the $O T$ and $T T$ scenario. In the first setup we estimated a CPWLF for each antenna. In the second setup we estimated common ZWD parameters for the twin stations. We call the solutions with combined ZWD parameters OTc and TTC.

We evaluated the different scenarios by comparing the estimated parameters with the true values. The differences between the estimated and the true value of UT1 and the ZWD are denoted with $\varepsilon_{\mathrm{UT} 1}$ and $\varepsilon_{\mathrm{ZWD}}$, respectively. For each scenario we calculated the error in UT1 $\varepsilon_{\mathrm{UT} 1}$ in all sessions and computed its mean and the standard deviation. The results are summarized in Tab. 2. Using the broadband instead of the legacy S/X

\footnotetext{
${ }^{1}$ vgosDB quality code larger or equal to five

2 The station clock in Wettzell was fixed, the other was estimated with a polynom of order two. We estimated one UT1 offset and for each station a ZWD offset.
} 


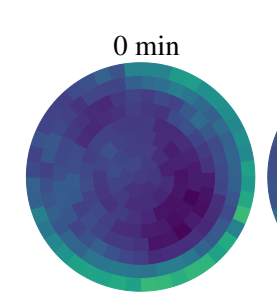

$12 \mathrm{~min}$
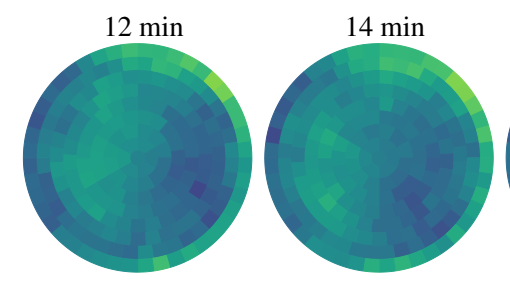

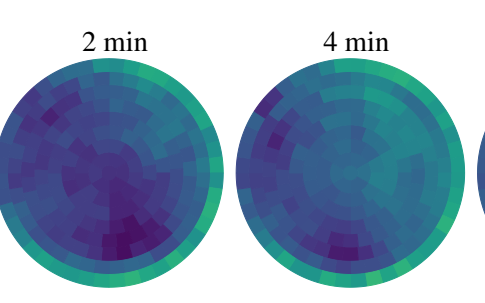

$16 \mathrm{~min}$

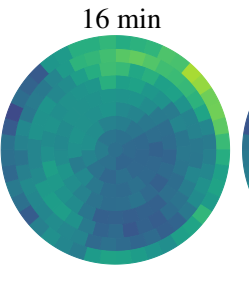

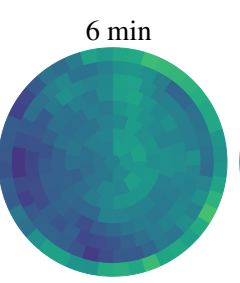

$18 \mathrm{~min}$

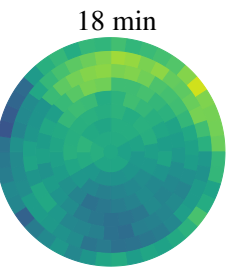

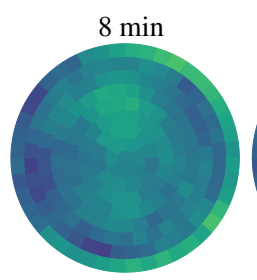

$20 \mathrm{~min}$
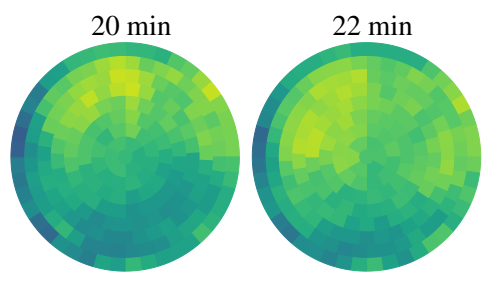

EZWD [mm]

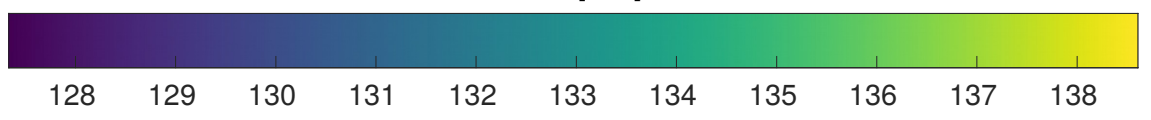

Fig. 2: Example of a simulated reference troposphere: Each sky plot represents the troposphere at one point of time. The time between two subsequent sky plots is two minutes, although the troposphere is computed with a temporal resolution of one minute. The sky plots are aligned to the north (top of this page) and the center cell corresponds to an elevation of 90 degrees. In this example, the wind is blowing from north-east direction.

Table 2: Mean and standard deviation of the error in UT1 w.r.t. all sessions. The original scenario is a simulation of the schedules that were used for the (observed) INT1 and INT2 sessions and analyzed with the standard intensive setup.

\begin{tabular}{lrr} 
scenario & mean $\varepsilon_{\mathrm{UT} 1}(\mu \mathrm{s})$ & std $\varepsilon_{\mathrm{UT1}}(\mu \mathrm{s})$ \\
\hline original & -0.5 & 11.0 \\
\hline$Z T$ & -0.2 & 8.3 \\
\hline OT & 0.4 & 6.2 \\
OTc & 0.5 & 6.2 \\
\hline$T T$ & 0.1 & 4.2 \\
$T T c$ & 0.1 & 4.2
\end{tabular}

setup (first row vs. second row) the standard deviation of the error in UT1 decreases by almost a factor of $3 \mu \mathrm{s}$. With each additional twin telescope the scatter is further decreased. In the TT scenario the standard deviation of the error in UT1 is reduced to $4 \mu$ s. Combining ZWD parameters, however, has no significant effect on the estimation of UT1.

We computed the statistics of the error of the ZWDs in the same way. The estimated ZWDs were compared with the ZWDs in the center cell of the reference troposphere. The results are given in Tab. 3. The standard de-
Table 3: Mean and standard deviation of the error for the ZWDs of each station w.r.t. all sessions. The refractive index structure constants for the involved stations are give in the last row.

\begin{tabular}{|c|c|c|c|c|c|c|}
\hline \multicolumn{7}{|c|}{ mean $\varepsilon_{\mathrm{ZWD}}(\mathrm{ps})$} \\
\hline scenario & Wn & Ws & $\mathrm{Kk}$ & Is & On & Os \\
\hline$Z T$ & -0.06 & - & -0.24 & -0.60 & - & - \\
\hline$O T$ & 0.09 & 0.33 & -0.44 & -0.15 & - & - \\
\hline OTC & \multicolumn{2}{|c|}{0.14} & -0.53 & -0.16 & - & - \\
\hline$T T$ & -0.30 & 0.09 & -0.53 & 0.22 & -0.21 & 0.17 \\
\hline$T T c$ & \multicolumn{2}{|c|}{-0.10} & -0.57 & 0.17 & \multicolumn{2}{|c|}{0.03} \\
\hline
\end{tabular}

\begin{tabular}{l|cccccc}
\multicolumn{7}{c}{ std $\varepsilon_{Z W D}(\mathrm{ps})$} \\
& $\mathrm{Wn}$ & $\mathrm{Ws}$ & $\mathrm{Kk}$ & $\mathrm{Is}$ & On & Os \\
\hline$Z T$ & 9.3 & - & 17.0 & 11.9 & - & - \\
\hline$O T$ & 8.5 & 9.0 & 16.6 & 11.4 & - & - \\
$O T c$ & 8.1 & & 16.6 & 11.4 & - & - \\
\hline$T T$ & 8.6 & 8.7 & 15.0 & 10.3 & 7.0 & 6.9 \\
$T T c$ & 8.0 & 15.0 & 10.4 & 6.1 & \\
\hline & \multicolumn{7}{|c}{ structure constant $C_{n}\left(10^{-7} \mathrm{~m}^{-\frac{1}{3}}\right)$} \\
& 0.94 & 2.30 & 1.46 & 0.72 &
\end{tabular}

viation of the error of the ZWDs decreases slightly by introducing one twin station (about a half picosecond). 


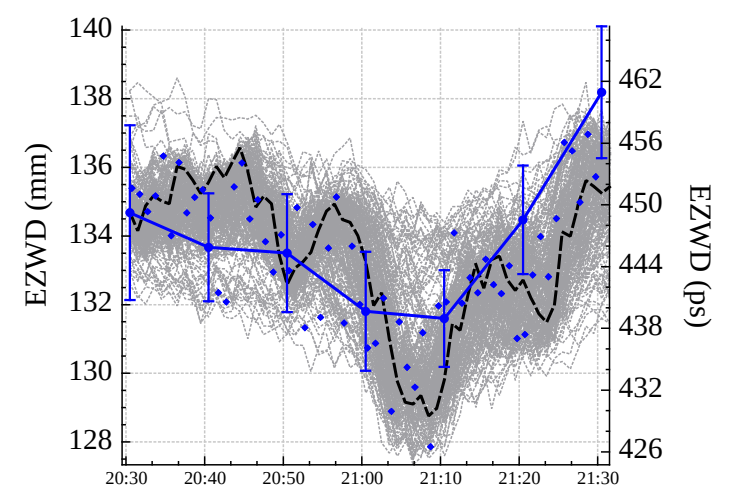

Fig. 3: Illustration of the reference troposphere at one station for one session (see Sec. 4 for a description).

This applies also to the non-twin stations. By estimating common ZWDs parameters the standard deviation is roughly decreased by one picosecond. Furthermore, there is a strong correlation between the standard deviation of the error and the refractive index structure constant (see last row of Tab. 3).

In case of a rotational symmetric atmosphere the EZWD is independent of the elevation and the azimuth of the observations. This case is illustrated with a black dashed line in Fig. 3, which corresponds to the center cell of the reference troposphere. But when considering turbulence, the EZWD depends on azimuth and elevation and cannot be illustrated with a single line only. Each gray dotted line in Fig. 3 corresponds to the EZWD in one of the reference troposphere's cells. The difference between the true EZWDs (blue diamonds) and the estimated ZWDs (solid blue line) can reach several millimeters. A more sophisticated troposphere model appears necessary to model the EZWDs more accurately.

\section{Conclusions}

Introducing VGOS twin stations into INT sessions leads to more observations with large east-west component, providing improved estimations of the solar time UT1. Our simulations show that the standard deviation of the error in UT1 decreases from $11 \mu \mathrm{s}$ using the legacy S/X setup to $4 \mu$ s using two VGOS twin stations with a broadband setup. The ZWD esti- mation is only slightly improved (some picoseconds). To improve the estimation of the EZWD, a better troposphere model with more parameters appears necessary. Twin stations lead to more observations at the same observatory, and thus will allow in the future a more complex troposphere modeling.

\section{References}

Altamimi Z, Rebischung P, Métivier L, Collilieux X (2016). ITRF2014: A new release of the International Terrestrial Reference Frame modeling nonlinear station motions. Journal of Geophysical Research: Solid Earth, 121(8), 61096131.

Beckers B, Beckers P (2012). A general rule for disk and hemisphere partition into equal-area cells. Computational Geometry, 45(7), 275-283.

Böhm J, Werl B, Schuh H (2006). Troposphere mapping functions for GPS and very long baseline interferometry from European Centre for Medium-range Weather Forecasts operational analysis data. Journal of Geophysical Research, 111, B02406.

Corbin A, Niedermann B, Nothnagel A, Haas R, Haunert J-H (2019). VLBI Scheduling with an Approach from Combinatorial Optimization. Journal of Geodesy, in preparation.

Fey A L, Gordon D, Jacobs C S, Ma C, Gaume R, Arias E, Bianco G, Boboltz D, Böckmann S, Bolotin S, et al. (2015). The second realization of the international celestial reference frame by very long baseline interferometry. The Astronomical Journal, 150(2), 1-16.

Gipson J (2018). Sked - VLBI Scheduling Software. Technical report, NASA Goddard Spaceflight Center.

Halsig S, Corbin A, Iddink A, Jaron F, Schuber T, Nothnagel A (2017). Current Development Progress in ivg::ASCOT. A new VLBI Analysis Software. In R. Haas \& G. Elgered (Eds.), Proceedings of the 23rd Meeting of the European VLBI Group for Geodesy and Astrometry Working Meeting, Gothenburg, Sweden, May 2017 (pp. 167-171).

Nilsson T, Haas R (2010). Impact of atmospheric turbulence on geodetic very long baseline interferometry. Journal of Geophysical Research: Solid Earth, 115(B3).

Petit G, Luzum B (2010). IERS Conventions (2010). IERS Technical Note 36, International Earth Rotation and Reference Systems Service, Verlag des Bundesamtes für Kartographie und Geodäsie Frankfurt am Main.

Petrachenko B, Niell A, Behrend D, Corey B, Böhm J, Charlot P, Collioud A, Gipson J, Haas R, Hobiger T, et al. (2009). Design Aspects of the VLBI2010 System-Progress Report of the IVS VLBI2010 Committee. Technical report, NASA Goddard Space Flight Center.

Schnell D (2006). Quality aspects of short duration VLBI observations for UT1 determinations. PhD thesis, Institut für Geodäsie und Geoinformation, University of Bonn.

Williams H P (2013). Model Building in Mathematical Programming, volume 5. John Wiley \& Sons. 